\title{
Review
}

\section{Huntingtin Interactions with Membrane Phospholipids: Strategic Targets for Therapeutic Intervention $?^{1,2}$}

\author{
Kimberly B. Kegel-Gleason* \\ Department of Neurology, Massachusetts General Hospital and Harvard Medical School, Charlestown, MA, USA
}

\begin{abstract}
The Huntington's disease gene encodes the protein huntingtin (Htt), a soluble protein that largely distributes to the cytoplasm where about half the protein is found in association with membranes. Early studies on Huntington's disease patients suggested changes in membrane phospholipids. Furthermore, changes in phospholipid biosynthetic enzymes have been found in HD cell models using genetic methods. Recent investigations prove that $\mathrm{Htt}$ associates with membranes by direct interactions with phospholipids in membranes. Htt contains at least two membrane binding domains, which may work in concert with each other, to target to the appropriate intracellular membranes for diverse functions. Htt has a particular affinity for a specific class of phospholipids called phosphatidylinositol phosphates; individual species of these phospholipids propagate signals promoting cell survival and regulating changes in morphology. Mutant $\mathrm{Htt}$ fragments can disrupt synthetic phospholipid bilayers and full-length mutant Htt shows increased binding to numerous phospholipids, supporting the idea that mutant Htt can introduce pathology at the level of phospholipid interactions. There is a great potential to develop therapeutic agents since numerous enzymes regulate the both the biosynthesis/metabolism of lipids and the post-translational modifications of Htt that direct membrane interactions. Understanding the relationship of Htt with membrane phospholipids, and the impact of mutant Htt on membrane-related functions and lipid metabolism, may help identify new modes of therapeutic intervention for Huntington's disease.
\end{abstract}

Keywords: Huntingtin, phospholipids, fatty acids, glycerophospholipids, membrane, phosphatidylinositol phosphate

\footnotetext{
${ }^{1}$ This research was originally published in the Journal of Biological Chemistry. Kegel KB, Sapp E, Yoder J, Cuiffo B, Sobin L, Kim YJ, Qin ZH, Hayden MR, Aronin N, Scott DL, Isenberg G, Goldmann WH, Difiglia M. Huntingtin associates with acidic phospholipids at the plasma membrane. J Biol Chem. 2005; 280:36464-36473. (C) the American Society for Biochemistry and Molecular Biology.

${ }^{2}$ Reprinted from Biophysical Journal, Vol 105 / August, M Michalek, ES Salnikov, and B Bechinger, Structure and Topology of Huntingtin 1-17 Membrane Anchor by a combined Solution and Solid-State NMR Approach., 699-710., Copyright (2013), with permission from Elsevier.

${ }^{*}$ Correspondence to: Kimberly B. Kegel-Gleason, Ph.D., Department of Neurology, Massachusetts General Hospital and Harvard Medical School, MIND, 114, 16th Street, Room 2001, Charlestown, MA 02129, USA. Tel.: +1 617724 8754; E-mail: kkegel@partners.org.
}

\section{THE HUNTINGTON'S DISEASE PROTEIN}

The Huntington's disease (HD) gene (IT-15 or HTT) encodes the protein huntingtin $(\mathrm{Htt})[1]$, which is ubiquitously expressed in postnatal mammalian cells and enriched in neurons [2-5]. Htt is an essential protein for many species. Null mutations in mice created by homologous recombination results in embryonic lethality at day 7 and conditional inactivation of $\mathrm{Htt}$ during development or postnatal inactivation causes testicular degeneration and selective neurodegeneration [6-10]. These studies indicate a highly important function for Htt.

Human Htt is a soluble protein composed of 3144 amino acids that largely distributes to the cytoplasm, 


\section{Huntingtin}

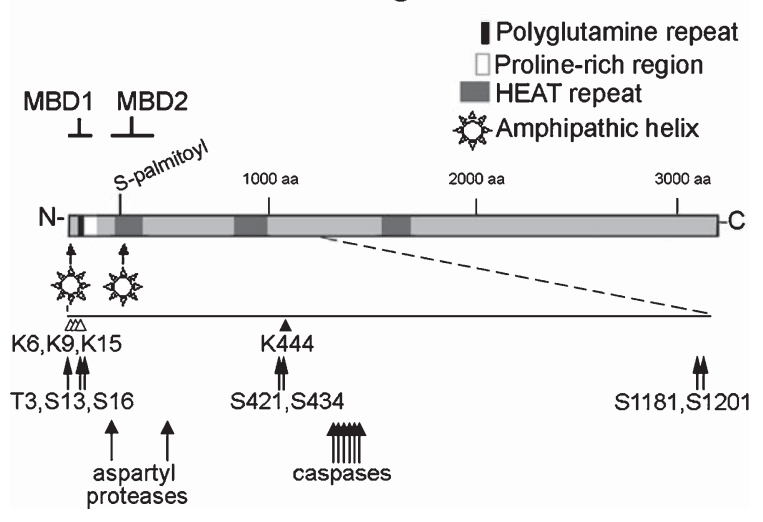

Fig. 1. Features of huntingtin $(\mathrm{Htt})$. Black shaded area indicates polyglutamine tract, which is abnormally expanded in HD. White area indicates a large polyproline region. HEAT repeats are shown in grey [45]; Ten repeats span three separate regions. Additional HEAT repeats have been detected throughout Htt (up to 88 repeats) using low threshold modeling [81]. MBD1, Membrane Binding Domain that is regulated by polyglutamine expansion exists between 1-89 [14, 43, 48]. MBD2, major membrane binding domain from aa 172-372, identified using deletion analysis [23]. The locations of amphipathic helices, which contribute to membrane binding, are indicated at 1-17 and 229-246 (yellow stars). Additional MBDs may yet be identified. Htt also can be palmitoylated at aa 214 [58]. Sites for post-translational modifications for phosphorylation/SUMOlation/ubiquitination (open arrowheads), acetylation (closed arrow head), and phosphorylation (small arrows) are indicated below. Large arrows show aspartyl protease and caspase cleavage sites in $\mathrm{Htt}$.

where about half the protein is found in association with membranes [2, 11, 12]. A CAG repeat is translated into a polyglutamine $(\mathrm{Q})$ tract near the N-terminus of Htt. Expansion beyond a threshold of 36 repeats causes HD (Fig. 1). Mutant Htt distributes to the same intracellular location as wild-type Htt including the plasma membrane, endosomes, the endoplasmic reticulum and synaptic sites [11-15]. Thus, mutant $\mathrm{Htt}$ is poised to disrupt normal Htt function at membranes and other membrane-related processes. Several membrane-associated functions have been ascribed to $\mathrm{Htt}$ although the exact molecular mechanisms are still being investigated. Htt can function to facilitate microtubule-dependent vesicle transport and may also help vesicles "decide" when to move from a microtubule to an actin filament and vice versa (reviewed by [16]. Studies on cells null for Htt implicate functions in adhesion [17], motility [18-20], Rab11-dependent vesicle budding [21], and cholesterol and energy homeostasis [18, 22]. Htt may act as a molecular scaffold for coordination of membrane and cytoskeletal communication $[16,23]$ and to support growth factor signaling complexes [24, 25].
Despite its prominent membrane localization, relatively little is known about the relationship of $\mathrm{Htt}$ with lipids and the role of lipids in HD. Microarray analysis of HD cells suggested transcriptional deregulation of the cholesterol pathway reduced cholesterol levels have been shown in numerous HD models (reviewed in detail elsewhere $[18,26])$. Changes in the ganglioside GM1, which is found in membrane rafts and affects signaling pathways, have also been found and ascribed to altered transcription of metabolic enzymes; treatment with GM1 provides protection an HD cellular model $[27,28]$. In this review, I will focus on the relationship of Htt with membrane phospholipids and known changes in HD.

\section{FATTY ACIDS AND GLYCEROPHOSPHOLIPIDS IN HD}

Glycerophospholipids or phospholipids are composed of fatty acids with hydrophobic acyl chains covalently adjoined to a glycerol backbone together with a polar head group. Phospholipids are amphipathic molecules that create bilayers and are major constituents of cellular membranes. Major phospholipid subclasses exist defined by their head group that are important for mammalian membranes: phosphatidic acid (PA), phosphatidylcholine (PC), phosphatidylethanolamine (PE), phosphatidylglycerol (PG), phosphatidylinositol (PI) and phosphatidylserine (PS), Sphingomyelin (SM) and Cardiolipin (CL) (www.lipidmaps.org). Membranes of different organelles are composed of varied combinations of phospholipids. Differences in phospholipid composition also exist between the inner and outer leaflets of membranes. Membrane architecture and lateral mobility are affected by phospholipid composition, including acyl chain length and bond saturation, which in turn may affect signaling pathways and membrane associated functions. Saturated acyl chains (carbon chains with no double bonds) have straight structures which can pack tightly, whereas polyunsaturated acyl chains have distended structures preventing tight packing in the bilayer and promoting lateral mobility. Acyl chain identity can also have direct impacts on signaling outcomes. For instance, the polyunsaturated fatty acid arachidonic acid incorporates into PC, PE and PI. In the brain, arachidonic acid can be liberated by phospholipases to act as a second messenger and affect neurotransmission (reviewed by S. Chalon 2006 [29]). Dopamine 2-receptor stimulation can activate this pathway and may modulate neurotransmission 
[30]. Modulation of neurotransmission via phospholipids is hypothesized to affect cognition [31], which is an early symptom of HD.

Prior to identification of the HD mutation, the properties of membranes isolated from autopsy tissue of HD patients and from erythrocytes suggested pathological changes in membrane phospholipid composition. Lloyd and Davidson speculated that regulation of $\gamma$ amino butyric acid (GABA) binding to receptors was influenced by phospholipid regulation in HD. They found that membranes isolated from postmortem cerebellum of HD patients had an increased affinity $(\mathrm{Kd})$ for tritiated GABA compared those isolated from normal individuals, despite similar total number of binding sites; treatment of normal membranes with phospholipase $\mathrm{C}$ or Triton $\mathrm{X}-100$ recapitulated the effect observed in HD membranes, and addition of PE to membranes corrected binding of GABA in HD membranes to control levels, suggesting an effect on phospholipids [32]. Ellison et al. reported decreases in phosphoethanolamine and ethanolamine (metabolites of PE) in both HD and in Alzheimer's disease and proposed the notion that phospholipid metabolism was altered in HD [33]. Another study by Sakai et al. showed reduced mean levels of docosahexaenoic acid in erythrocyte membranes of HD patients versus controls [34]. In a small clinical study, Puri found an altered response of HD patients to a skin flush test in response to niacin, which suggested changes in fatty acid metabolism [35], since vasodilation in response to niacin is thought to depend on liberation of arachidonic acid from membranes and its conversion to Prostoglandin D2 [36, 37]. Thus, physicians and scientists have long hypothesized that changes in membrane phospholipids might cause pathology in HD patients [38].

Clinical and preclinical trials supplementing diet with fatty acids to alter or restore membrane composition have yielded mixed results. Studies in a transgenic mouse model of HD expressing exon 1 of human $\mathrm{Htt}$ (R6/1 mice) showed that supplementation of a mix of essential fatty acids could improve motor symptoms, but did not alter disease course [39]. A study using oral ethyl-eicosapentaenoate (ethyl-EPA), a pro form of the n-3 essential fatty acid EPA, showed significant improvement in motor symptoms with no change in neurodegeneration in the YAC 128 mouse model of HD, which over-expresses full-length human Htt [40]. Clinical trials in human HD patients with ethyl-EPA showed stability or improvement of motor function in patients who adhered to the protocol (PP, or "per protocol") compared to placebo [41]; however, no significance was achieved with the larger cohort of "Intent-to-treat" patients and the study design was not intended to measure changes in motor function. In patients from the same study, reduced cerebral atrophy was also observed in the PP group compared to placebo [42]. In two follow up Phase III studies specifically designed to determine whether ethyl-EPA improves motor function after 6 months treatment, no significant difference between the placebo and treatment group was found [43, 44]; however, in the open label phase which followed for an additional 6 months, for patients in the treatment group the total motor score did not change from their initial baseline score at the beginning of the trial, whereas for patients in the placebo group total motor scores were worse [43]. While the results at 12 months suggest some efficacy, more than one third of the placebo group dropped out of the study at 6 months and the study was not powered to detect changes as small as those measured at 12 months, so no positive conclusions can be made. Although viewed as failed trials, these results do not exclude the possibility that treatment with a different fatty acid or mix of fatty acids, or longer term treatment with ethyl-EPA, might have disease-modifying effects in HD.

Metabolism of phospholipids and fatty acids has also been implicated in cell and animal models of HD. Decreased levels of transcription for enzymes in the biosynthetic pathway for triglycerides and phospholipids were found in cells expressing mutant $\mathrm{Htt}$ $[38,45]$. Changes in lipid/sterol/lipoprotein pathways were found in a genome-wide expression analysis of an allelic series of mouse embryonic stem cells expressing endogenous $\mathrm{Htt}$ with increasing polyglutamine lengths; Htt null cells also showed changes in the lipid/sterol/lipoprotein pathway, although a different set of genes were changed [22]. Mutant Htt increases the sensitivity of the inositol $(1,4,5)$ triphosphate (IP3) receptor to IP3, resulting in altered intracellular calcium homeostasis [46]. IP3 is produced together with the signaling molecule diacylglycerol (DAG) from PI $(4,5)$ bisphosphate by phospholipases. A recent study by Ellerby and colleagues also implicates diacylglycerol kinase (DGK) $\varepsilon$, which catalyze conversion of DAG to PA, in HD [47]. Using a screen of small molecule kinase inhibitors, they identified DGK inhibitor II as a molecule that could suppress mutant $\mathrm{Htt}$ induced toxicity in a cell model. Levels of DGK $\varepsilon$ in particular were increased in R6/2 HD transgenic mouse striatum and siRNAs targeting DGK $\varepsilon$ could decrease toxicity in their cell model [47].

New methods of mass spectrometry together with generation of lipid standards developed by small 
biotech companies have revolutionized the ability of chemists to detect and quantify lipid species from complex mixtures. Though still difficult due to the large number of molecular species present in living organisms, several groups have undertaken the task of identifying novel changes in levels of phospholipids and related metabolites between control and HD patients, as well as between wild-type and HD mice models. It will then be a challenge to determine whether any measured changes are caused directly by the HD mutation or are downstream sequelae, and ascertain whether therapeutic intervention is possible or useful.

\section{HUNTINGTIN STRUCTURE AND MEMBRANE BINDING DOMAINS}

Htt contains two membrane binding domains found within the first 500 amino acids capable of binding directly to phospholipids and targeting Htt to cellular membranes (Fig. 1) [14, 23, 48, 49]. Extensive deletion analysis within Htt 1-969 defined aa171-373 as a major membrane binding domain for wild-type Htt (Fig. 1, MBD2) [23]. Expression of Htt 1-287 which contains half of this region targets almost exclusively to the plasma membrane when detected using immunofluorescence [23]; expression of Htt 1-969 targets to plasma membranes at clathrin coats, endosomes, multivesicular bodies, lysosomes and autophagosomes when detected using immunogold electron microscopy [11]. Computational structural predictions suggest that this domain is largely alpha helical, precisely matching the HEAT repeats first defined by Andrade and Bork [50], and bears an amphipathic helix. The sequence containing the helix was shown to insert into phospholipid bilayers empirically using differential scanning calorimetry (Fig. 2A-C ${ }^{1}$ ) [23]. Electrostatic mapping indicated the area encompassing amino acids 172372 emanates a large positive charge (Fig. 2D ${ }^{1}$ ). We determined using lipid overlays that $\mathrm{Htt}$ bound preferentially to acidic (negatively charged) phospholipids, consistent with the presence of a cationic binding domain [23]. Importantly, we demonstrated that immunopurified Flag-tagged Htt directly bound synthetic large unilamellar vesicles (LUVs) composed of PC:PE and phosphatidylinositol 3,4-bisphosphate $(\mathrm{PI}(3,4) \mathrm{P} 2)(5: 4: 1$ molar ratios), supporting the contention that Htt binds to membranes through direct interactions with phospholipids [49].

Another membrane binding domain exists within 1-89aa (or exon1) of Htt (Fig. 1, MBD1). Studies show that Htt residues 1-17 and 1-89 (exon1) fused to GFP or GST, respectively, can target to synthetic membranes [14, 48, 49, 51, 52]. Structural predictions and point mutations showed that aa1-17 of $\mathrm{Htt}$ also form an amphipathic helix, which can target Htt 1-89 to ER membranes and autophagic vacuoles in cells [14], and to mitochondrial, Golgi and ER membranes [53]. Changes in helical content monitored by circular dichroism were observed with $\mathrm{Htt}$ 1-17 incubated with LUVs of PC or PC:PS (7:3 molar ratio), and blocked by mutation of a methionine at aa 8 to proline (M8P) [14], supporting the notion that Htt directly interacts with phospholipids. Wanker and colleagues investigated interactions of purified GST-Htt 1-89 with lipid vesicles of various compositions [48]. They found that both soluble GST-Htt1-89 with normal and expanded polyglutamines could bind and perturb LUVs composed of brain lipids, and that the proline region adjacent to the polyglutamine stretch (Fig. 1) was important for the interaction with lipids [48]. Recent studies using atomic force microscopy to monitor aggregation and vesicle permeation confirm that Htt 1-17 inserts into synthetic lipid membranes and show that the polyproline region can modulate its behavior [51, 52]. Using solid state nuclear magnetic resonance (NMR), Michalek et al. demonstrated that Htt1-17 converts from random coil in aqueous solution to an alpha-helical structure when associated with lipid bilayers, and aligns almost parallel to the surface with a tilt angle of $75^{\circ}$ [51]. Further studies using solution- and solid-state NMR to determine the high resolution structure of $\mathrm{Htt}$ 1-17 in phosphocholine micelles and phospholipid bilayers indicate that $\mathrm{Htt}$ K6-F17 adopt an alpha-helical conformation upon binding to phospholipids (Fig. $3^{2}$ ) [54]. X-ray crystallography of Htt 1-89 with a wild-type glutamine tract also showed Htt 1-17 forms an alpha-helix as a crystal [55]. In agreement with these studies, we also found that wild-type Htt 1-102 containing MBD1 can interact with phospholipids and GST-Htt1-89 could insert into lipid bilayers containing PS or PG [49, 56]; however, the affinity for cellular membranes or LUVs is much lower for Htt 1-102 when compared directly to $\mathrm{Htt}$ 1-287 aa, or compared to longer $\mathrm{Htt}$ fragments containing the full MBD2. Consistent with this finding, Michalek and colleagues found that Htt1-17 a) had reduced membrane partitioning as measured by transition from random coil to helical conformation using circular dicroism and b) did not permeabilize synthetic lipid membranes when tests were performed with compositions that closely mimicked cell membranes [51]. In this study, syn- 
A

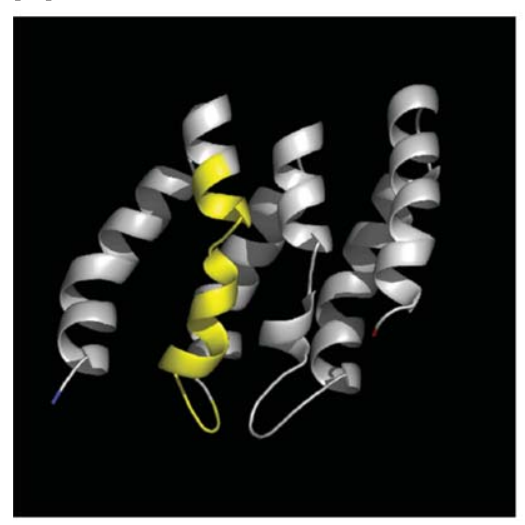

B

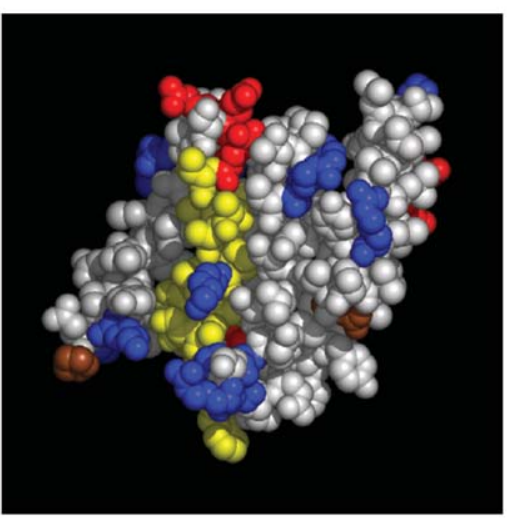

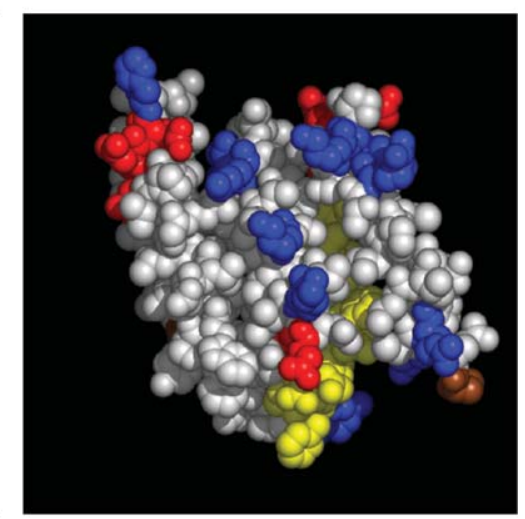

C

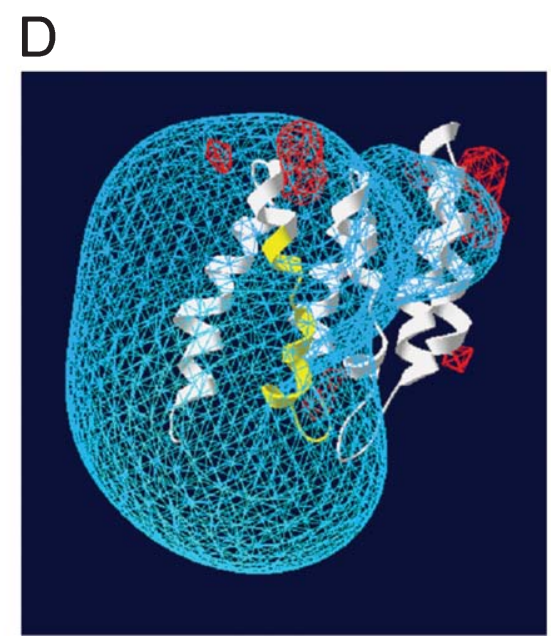

Fig. 2. A, Three-dimensional model for htt residues 201-327 based on homology modeling with the crystalline coordinates from the Protein Phosphatase 2A PR65/A. See original article for color. The consecutive 3 HEAT repeats are each composed of paired alpha-helices. The second helices of the first 2 repeats are interrupted in their mid sections producing a kink in the overall alignment. The region that can insert into synthetic lipid vesicles (residues 229-246) is colored yellow, the amino-terminus is in blue, and the carboxyl-terminus is colored red. B, Space-filling representations of (A) demonstrates the distribution of charge groups. Negatively charged residues (D, E) are colored red, positively charged amino acids are colored blue $(\mathrm{H}, \mathrm{K}, \mathrm{R})$ and the amino/carboxyl termini are indicated in brown. The model on the left is oriented identically to that in (A) whereas the right model has been rotated 180 degrees around the vertical axis. C, Helical wheel representation of htt segment 229-241 as an amphipathic helix. Although shown here as a continuous helix, homology modeling with the HEAT repeats of the protein phosphatase 2A PR65/A suggests that only htt residues 229-231 and 235-241 are true alpha-helix (as in A). Intercalated residues 232-234 form a distorted helix-type structure that may be conformationally flexible. The dashed line demarcates the hypothetical boundary between lipid and cytosol; hydrophobic residues (black) on the top surface of the helix would interact with the hydrophobic acyl chains of the phospholipids, while neutral, charged (blue) and polar residues (green) would interact with the polar headgroups of phospholipids and aqueous cytosol. The wheel was drawn with the assistance of WinPep [82]. D, Electrostatic potential map for htt residues 201-327 with residues 229-246 shown in yellow. A large distribution of positive potential may account for htt's preference for anionic phospholipids. The potential map was calculated with Deep View - The Swiss-PdbViewer using the Coulomb method with a solvent dielectric constant of 80 . The map is contoured at $-1.80 \mathrm{kT} / \mathrm{e}(\mathrm{red})<0.0 \mathrm{kT} / \mathrm{e}$ (white) $<1.80 \mathrm{kT} / \mathrm{e}$ (blue) [83]. ${ }^{1}$

thetic unilamellar vesicles composed of 1-palmitoyl2-oleoyl-sn-glycero-3-phosphocholine/1-palmitoyl-2oleoyl-sn-glycero-3-phospho-L-serine (POPC/POPS) (75:25 molar ratio) were used for circular dicroism and dye release assays. When cholesterol was added to the POPC/POPS vesicles, dye release and membrane partitioning were significantly reduced. Since choles- terol is a major component of plasma membranes, this may explain why the two studies using $\mathrm{Htt}$ 1-89 did not find plasma membrane targeting [14, 53]. As discussed above, larger Htt fragments containing MBD2 and full length $\mathrm{Htt}$ can localize to the plasma membrane, implicating MBD2 in targeting to cholesterol-enriched membranes. Truants and colleagues have reported 
Table 1

Characteristics of phospholipids bound by wild-type $\mathrm{Htt}$

\begin{tabular}{|c|c|c|c|c|}
\hline Lipid & $\mathrm{PI}(3,4) \mathrm{P} 2$ & $\mathrm{PI}(3,5) \mathrm{P} 2$ & $\mathrm{PI}(3,4,5) \mathrm{P} 2$ & Cardiolipin \\
\hline Membrane type & $\begin{array}{l}\text { PM, Early endosomes, } \\
\text { Multivesicular bodies }\end{array}$ & Late endosomes & $\mathrm{PM}$ & $\begin{array}{l}\text { Mitochondria inner } \\
\text { membrane }\end{array}$ \\
\hline $\begin{array}{l}\text { Associated cell } \\
\text { functions }\end{array}$ & $\begin{array}{l}\text { Signaling (Growth Factor, } \\
\mathrm{H}_{2} \mathrm{O}_{2} \text { ), Endocytosis }\end{array}$ & $\begin{array}{l}\text { Lysosomes, } \\
\text { Autophagy }\end{array}$ & $\begin{array}{l}\text { Signal Transduction (Growth } \\
\text { Factor- PI3K) }\end{array}$ & $\begin{array}{l}\text { General membrane } \\
\text { component apoptosis, } \\
\text { cytochrome c release }\end{array}$ \\
\hline
\end{tabular}
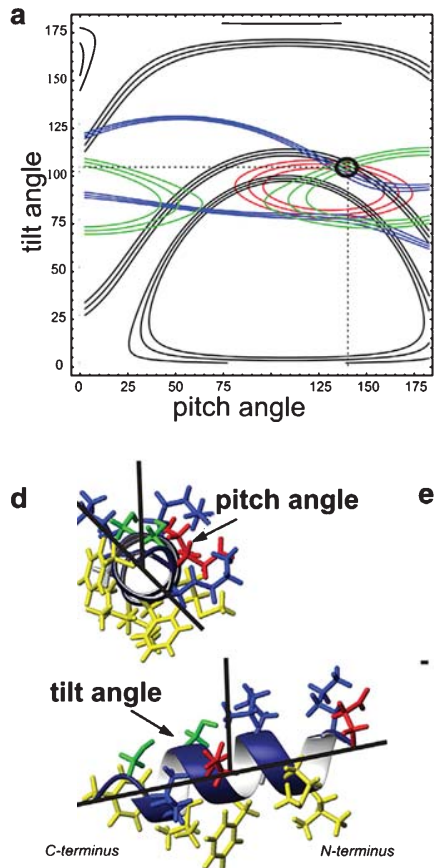

e
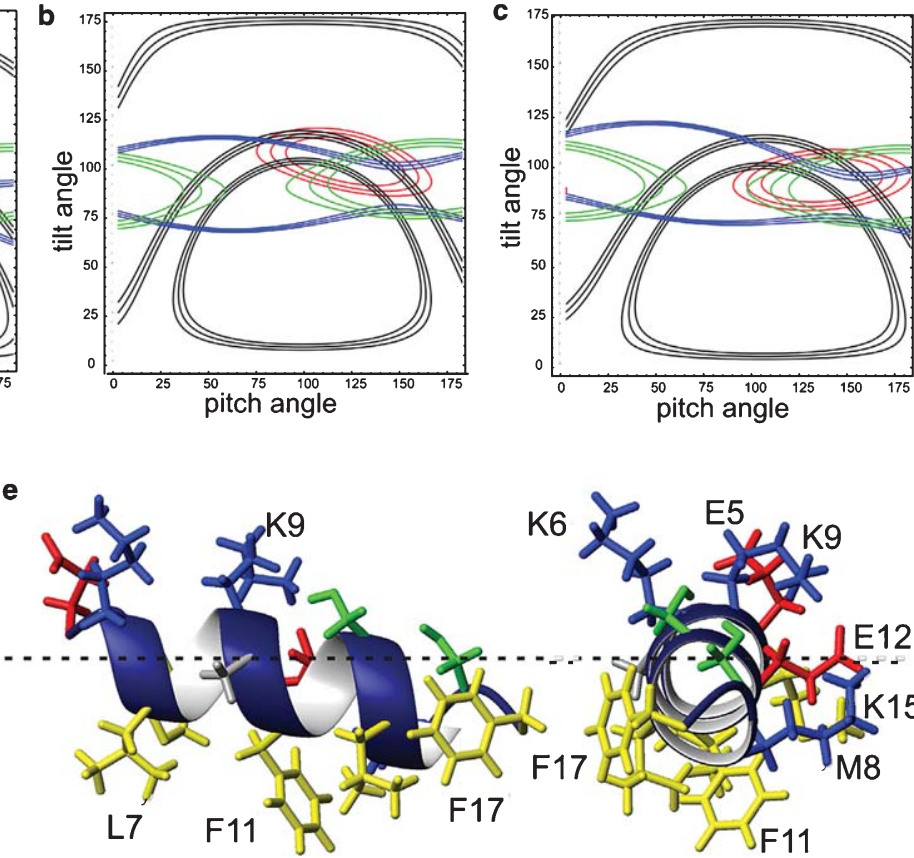

K6

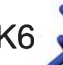

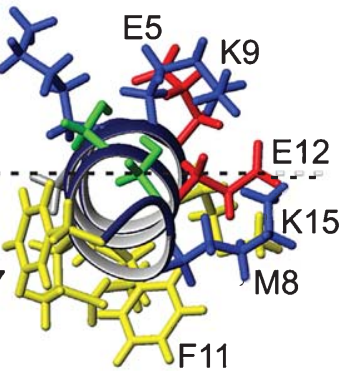

f

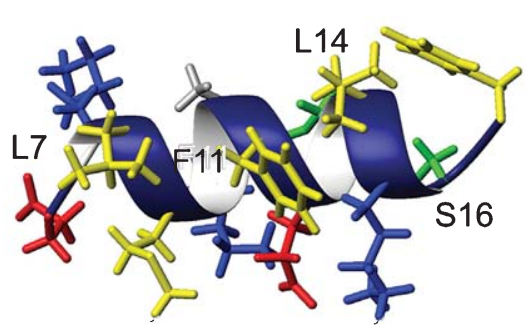

View from the membrane interior

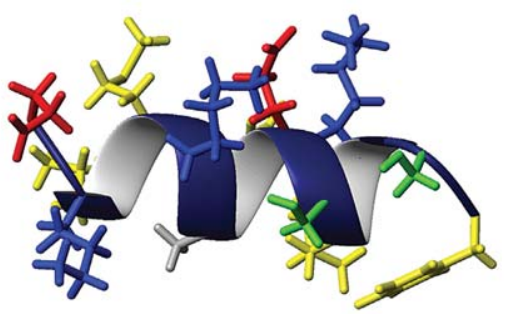

View from the aqueous side

Fig. 3. Taken from Michalek et al. [54]. Angular restrictions obtained from solid-state NMR spectra of huntingtin 1-17 reconstituted in oriented POPC bilayers. (a) The possible alignments of the low-energy conformer 3 obtained by solutionNMR in the presence of DPC micelles, structure calculation, and refinement are represented by their helical tilt and the rotational pitch angles. The solid black lines represent angular pairs that agree with the experimental $2 \mathrm{H}$ quadrupolar splitting obtained from $2 \mathrm{H} 3-\mathrm{Ala}-10(1152.5 \mathrm{kHz})$, the $15 \mathrm{~N}$ chemical shifts of $15 \mathrm{~N}-\mathrm{Leu}-7$ (red; 71.552 .5 ppm), 15N-Phe-11 (green; 78.553 ppm), and 15N-Phe-17 (blue; 8851.7 ppm) (cf. Fig. 4, a, c, e, and g). The error bars correspond to the line width at half-height. The tilt/rotational pitch angular pair is circled where all experimental data agree. In (b) the related analysis performed with the lowest energy structure 1 and in (c) with the average of the 20 low-energy conformations are depicted. Peptide dynamics were taken into account by wobbling motions of the helix (10_ Gaussian distribution) as well as pitch angle fluctuations around the helix long axis (18_). (d) Exhibits the pitch angle and tilt angle definitions with some complimentary views to those depicted in (e) and (f). (e) Structural details of residues 5-17 are shown when viewed from the side or from the carboy-terminus, or (f) when viewed from the membrane interior (left) or the aqueous side (right). The hydrophobic residues are shown in yellow, alanine in gray, serines in green, glutamates in red, and lysines in blue. See original article for color. ${ }^{2}$ 
that M8P mutation in MBD1 is sufficient to disrupt membrane localization of exogenous full-length $\mathrm{Htt}$ in HEK cells, suggesting this region is a master regulator for Htt. One caveat to this experiment is that only the ratio of cytoplasmic to total fluorescent signal was quantified. No biochemical subcellular fractionations were performed for the exogenously expressed proteins in HEK cells; thus, one cannot determine whether proteolysis proximal to MBD2 occurred liberating a fragment capable of diffusing into the nucleus. A change in fluorescence could also be due to a shift of soluble cytoplasmic Htt to the nucleus. The M8P mutation may disrupt the nuclear export signal function of N1-17 [47, 57], causing increased nuclear localization of normally soluble Htt without affecting the membrane-localized pool of Htt. Furthermore, mutation of S13 and S16 to alanine or aspartate in fulllength $\mathrm{Htt}$ in BACHD mouse brain showed no change in subcellular distribution assessed by IHC and by subcellular biochemical fractionations which included nuclear, mitochondrial and microsomal fractions [58]. Thus, while Htt1-17 is a powerful domain targeting $\mathrm{Htt}$ exon1 protein (Htt1-89) to membranes, other domains in full-length Htt work in concert with or compete with Htt1-17. Additional MBDs may exist in regions distal to aa969 that have not yet been identified.

\section{Membrane targeting through binding to specific phospholipids}

Targeting to discreet sites on the plasma membrane or on specific organelles can occur through binding to specific lipids. Phosphatidylinositol phosphates (PIPs) are a set of anionic phospholipids which can serve as docking sites on membranes for specific proteins creating compartmentalized function [59-61]. PIPs are created by phosphorylation of PI at the 3,4 , or 5 positions on the inositol lipid head group. PIPs can be quickly created or metabolized by lipid kinases and phosphatases, facilitating rapid propagation and termination of signals. Lipid overlay experiments indicated that Htt binds multivalent PIPs, consistent with an electrostatic mode of association [23]. When tested using in vitro binding and sedimentation assays with synthetic LUVs to quantify affinity, the specific PIPs to which Htt associated in order of decreasing strength were: PI 3,4-bisphosphate (PI(3,4)P2), PI 3,5- bisphosphate (PI(3,5)P2), and PI 3,4,5-triphosphate (PI $(3,4,5) \mathrm{P} 3)$ [49]. These phospholipids are found in low abundance in membranes $(0.1 \%$ of total lipids) and localize to discreet intracellular locations to perform specialized functions (Table 1). Htt binds to PIPs that are prod- ucts of PI 3-kinases. There are several classes of PI 3-kinases which regulate numerous functions including cell survival, morphology changes, and membrane dynamics $[62,63]$. Htt mobilized to the plasma membrane after growth factor stimulation [49], which activates class $\mathrm{I}_{\mathrm{A}} \mathrm{PI} 3$-kinases producing $\mathrm{PI}(3,4) \mathrm{P} 2$ and $\mathrm{PI}(3,4,5) \mathrm{P} 3$ [63, 64]. Together, these results suggest that Htt may normally function in receptor tyrosine kinase/PI 3-kinases dependent signaling (Fig. 4). For instance, Htt could act as a scaffold to build multiprotein complexes at membrane surfaces to coordinate actin dependent neurite outgrowth. Htt may participate in other functions associated with particular phospholipids such as budding of endocytic vesicles from the plasma membrane or multivesicular bodies. Interestingly, Huntingtin Interacting Protein 1 (HIP1) [65, 66], which is involved in endocytosis and receptor tyrosine kinase stability, also binds PI(3,4)P2 and PI(3,5)P2 [67]. Htt may work in concert with HIP1 at membranes enriched in these PIPs, although Htt does not require HIP1 to target to membranes [23]. Future studies should illuminate these and other lipid dependent functions of Htt.

The highest affinity binding of Htt for any phospholipid measured was for cardiolipin [49]. However, cardiolipin is found on the inner mitochondrial membrane; whereas Htt1-89 has been localized to outer mitochondrial membranes [53], little, if any, $\mathrm{Htt}$ has been detected with inner mitochondrial membranes using immunofluorescence, biochemical methods or immuno-electron microscopy. Further investigations will be required to determine if $\mathrm{Htt}$ has a normal function associated with mitochondria or cardiolipin.

\section{Regulations of Htt membrane interactions}

Regulation of Htt association with membranes is still being explored but may occur through multiple post-translational mechanisms or through proteolysis of Htt (Fig. 1). Targeting and anchoring of Htt to specific membranes may be influenced by palmitoylation at cysteine 214 by the Htt interacting protein and palmitoyl acyl transferase, HIP14 [68, 69]. Wildtype Htt enhances the transferase activity of HIP14 and the related protein, HIP14 L, on target proteins including the synaptic protein SNAP25; mutant Htt interferes with enzymatic activity and interacts less well with HIP14. Indeed, loss of HIP14 or HIP14 L in mice results in a HD-like phenotype. Membrane interactions dependent on Htt 1-17aa could be modulated by phosphorylation near the amino terminus 


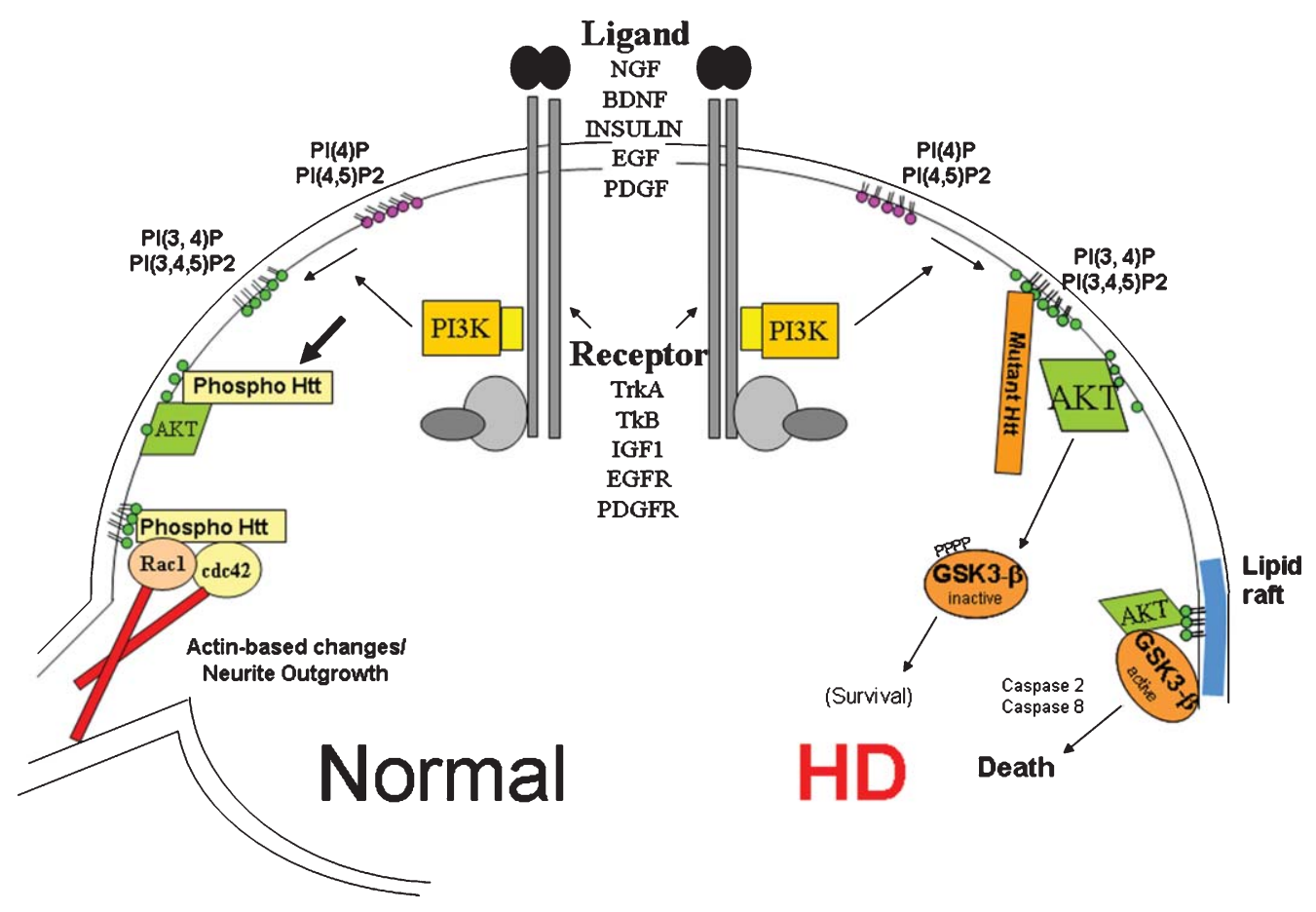

Fig. 4. Model for Htt recruitment by PIPs to the plasma membrane during growth factor signaling. Receptor tyrosine kinases are activated when bound by their ligand (growth factors), autophosphorylate and recruit the p85 regulatory subunit together with the p110 catalytic subunit of PI 3-kinase. Other proteins kinases such as ERKs are also recruited at independent sites. Using PI(4)P2 and PI(4,5)P2 as a substrate, PI 3-kinase creates $\mathrm{PI}(3,4) \mathrm{P} 2$ and $\mathrm{PI}(3,4,5) \mathrm{P} 3$ at the membrane. These PIPs provide high affinity binding sites on the inner membrane (cytosolic) surface for proteins such as Htt and AKT. Htt may act as a scaffold to build complexes that regulate signaling or subcortical actin remodeling and neurite outgrowth (left). Normal phosphorylation of Htt by AKT (left) is inhibited in HD (right). In the presence of Mutant Htt, active, unphosphorylated GSK3beta accumulates at rafts where it may activate cell death pathways and contribute to neuronal death (right).

[14]. Phoshorylation occurs in vivo at T3 and can modulate aggregation in Q140 HD mice, but effects on membrane localization have not been investigated [70]. Phosphorylation at S13 and S16 regulate further modification at K6, K9, K15 by acetylation, SUMOlation, and ubiquitination [71-73]. Phosphorylation at either S13 or S16 releases Htt1-89 from membranes and increases turnover; however, as discussed above, mutation of S13 and S16 to alanine or aspartate in full-length $\mathrm{Htt}$ in BACHD mouse brain did not change subcellular locale [58]. Phosphorylation at S421 by AKT and at S434, S1181, and S1201 by cdk5 [74-77] could also regulate membrane interactions through unknown MBDs or by regulating intramolecular interactions within Htt. Acetylation at Htt 444 targets Htt to autophagic membranes [78]. Htt is subject to proteolysis by numerous proteases including aspartyl proteases, which cleave between MBD1 and MBD2 and could act to separate function of the two MBDs, and caspases, which cleave distal to MBD2 and could liberate C-terminal region of Htt. Reduced affinity for membranes could also be achieved rapidly through modification of PIPs by lipid phosphatases, thus eliminating the high affinity-binding site for Htt. In summary, numerous enzymatic targets may exist that regulate Htt-membrane interactions. Future studies will be needed to determine if any are suitable for testing therapeutic intervention.

\section{POLYGLUTAMINE EXPANSION AND MEMBRANE DISRUPTION IN HD}

Polyglutamine expansion has profound effects on membrane binding of small exogenously expressed $\mathrm{Htt}$ fragments containing only MBD1. Mutant Htt1-102 showed increased binding to synthetic lipid vesicles [49], and GST-Htt1-89 with expanded polyglutamines showed increased insertion into lipid bilayers using differential scanning calorimetry [56]. In the study by Suopanki et al., LUVS composed of brain lipids could stimulate fibrillogenesis of mutant Htt 1-89, where-as LUVs composed of PC reduced insoluble aggregate formation [48]. Recent studies using atomic 
Table 2

Characteristics of phospholipids with increased binding of mutant $\mathrm{Htt}$

\begin{tabular}{|c|c|c|c|c|}
\hline Lipid & $\mathrm{PE}$ & $\mathrm{PI}(4,5) \mathrm{P} 2$ & $\mathrm{PI}(3,4,5) \mathrm{P} 2$ & Cardiolipin \\
\hline Membrane type & Most membranes & $\mathrm{PM}$ & $\mathrm{PM}$ & $\begin{array}{l}\text { Mitochondria inner } \\
\text { membrane }\end{array}$ \\
\hline $\begin{array}{l}\text { Associated cell } \\
\text { functions }\end{array}$ & $\begin{array}{l}\text { General membrane } \\
\text { component, Autophagy }\end{array}$ & $\begin{array}{l}\text { Endocytosis, Signal } \\
\text { transduction (PLC; } \\
\text { PI3K) }\end{array}$ & $\begin{array}{l}\text { Signal Transduction (Growth } \\
\text { Factor-PI3K) }\end{array}$ & $\begin{array}{l}\text { General membrane } \\
\text { component apoptosis, } \\
\text { cytochrome c release }\end{array}$ \\
\hline
\end{tabular}

force microscopy confirmed that aggregation of $\mathrm{Htt}$ exon1 was accelerated by membranes from purified brain lipids, and showed that lipid induced aggregation was polyglutamine length dependent [52]. Truant and colleagues reported a loss of ER targeting with mutant $\mathrm{Htt}$ and a concatenate increase in nuclear entry [14]. Disruption of lipid bilayers has been hypothesized to occur by a mechanism where-by extended polyglutamine tracts insert and form channels [79]. Whether this can occur when polyglutamine is in the context of Htt protein is unclear, but perturbations of phospholipid bilayers clearly occur when mutant Htt fragments are introduced.

Full-length endogenous mutant Htt from homozygous Q140 knock-in HD mouse brain cytosol has increased interactions with several phospholipids compared to wild-type $\mathrm{Htt}$ when tested using in vitro binding and sedimentation assays with synthetic lipid vesicles [49]. Of particular interest, mutant Htt showed significant binding to $\mathrm{PE}$, a major constituent of all membranes, and to PI 4,5-bisphosphate (PI $(4,5) \mathrm{P} 2)$ (Table 2); in contrast wild-type Htt does not bind appreciably to either of these phospholipids. Because PE is such an abundant membrane phospholipid, binding of mutant Htt to PE could affect lateral lipid mobility and raft formation, or prevent mutant $\mathrm{Htt}$ from localizing to normal membrane sites and compartmentalizing function. Increased binding to PE might also contribute to the membrane-related effects on GABA binding affinity observed in HD patients described above. Increased binding of mutant $\mathrm{Htt}$ to $\mathrm{PI}(4,5) \mathrm{P} 2$ could prevent normal binding of other protein PI effectors [61], or alter metabolism to the signaling molecules diacylglyceral (DAG) and Inositol $(1,4,5) \mathrm{P} 3$ by phospholipase C [60] . Ellerby and colleagues reported changes in levels of PI and PIP2 (likely PI(4,5)P2) containing stearoyl and arachidonoyl acyl chains $(38: 4)$ in a striatal cell model of HD [47]. Mutant Htt showed increased association with the signaling lipid PI $(3,4,5) \mathrm{P} 3$ compared to wildtype Htt [49]. In HD, this may result in altered PI 3-kinase dependent signaling downstream of growth factors such as BDNF. Interestingly, Htt is normally phosphorylated by AKT in response to growth factor stimulation and mutant Htt is less efficiently phosphorylated; Htt AKT proteins bind to $\mathrm{PI}(3,4,5) \mathrm{P} 3$ during growth factor stimulation (Fig. 4). One possibility is that inappropriate binding of mutant $\mathrm{Htt}$ to $\mathrm{PI}(3,4,5) \mathrm{P} 3$ sterically hinders its interaction with the catalytic site in AKT.

Endogenous mutant Htt is found at higher levels in primary neuron membrane raft fractions, as is GSK3beta, a signaling molecule that regulates cell death [80]. Raft fractions are thought to represent segregated membrane domains that are composed of a different set of phospholipids with a high percentage of saturated acyl chains, whereas non-raft fractions have a higher percentage of unsaturated acyl chains, and also contain cholesterol and GM1 and PI(3,4,5)P3. It is as yet unclear how mutant Htt alters GSK3-beta localization to rafts, however, HD primary neurons are protected by GSK3-beta inhibitors (Fig. 4) [80].

\section{CONCLUSIONS}

Membrane related changes were described as early biochemical features of HD and continue to be important avenues of study due to the prominent membrane location of Htt. Htt associates with membranes via direct interactions with phospholipids through at least two domains that may work in concert with each other to target to the appropriate intracellular membrane for diverse functions. Amino-terminal mutant Htt fragments can disrupt synthetic phospholipid bilayers and full-length mutant Htt shows increased binding to numerous phospholipids, supporting the idea that mutant Htt can introduce pathology at the level of phospholipid interactions. Current studies on phospholipid levels using mass spectrometry will determine whether Htt-phospholipid interactions also alter metabolism of lipids in HD. Future studies defining the regulation of Htt-phospholipid interactions will be crucial for elucidating molecular mechanisms of normal Htt function and may identify potential new targets for therapeutic interventions in HD. 


\section{ACKNOWLEDGEMENTS}

I am grateful to Xueyi Li for stimulating discussions regarding membrane association and function of Htt. I thank Ellen Sapp for her assistance with this manuscript. I also thank the editors of JHD for helpful input regarding the clinical trials discussed within. I am funded by CHDI Foundation.

\section{REFERENCES}

[1] HDCRG. A novel gene containing a trinucleotide repeat that is expanded and unstable on Huntington's disease chromosomes. The Huntington's Disease Collaborative Research Group. Cell. 1993;72:971-83.

[2] Difiglia M, Sapp E, Chase K, Schwarz C, Meloni A, Young $\mathrm{C}$, et al. Huntingtin is a cytoplasmic protein associated with vesicles in human and rat brain neurons. Neuron. 1995; 14:1075-81.

[3] Sharp AH, Loev SJ, Schilling G, Li SH, Li XJ, Bao J, et al. Widespread expression of Huntington's disease gene (IT15) protein product. Neuron. 1995;14:1065-74.

[4] Li SH, Schilling G, Young WS, 3rd, Li XJ, Margolis RL, Stine OC, et al. Huntington's disease gene (IT15) is widely expressed in human and rat tissues. Neuron. 1993;11:985-93.

[5] Strong TV, Tagle DA, Valdes JM, Elmer LW, Boehm K, Swaroop $\mathrm{M}$, et al. Widespread expression of the human and rat Huntington's disease gene in brain and nonneural tissues. Nat Genet. 1993;5:259-65.

[6] Nasir J, Floresco SB, O'Kusky JR, Diewert VM, Richman JM, Zeisler J, et al. Targeted disruption of the Huntington's disease gene results in embryonic lethality and behavioral and morphological changes in heterozygotes. Cell. 1995;81:81123.

[7] Duyao MP, Auerbach AB, Ryan A, Persichetti F, Barnes GT, McNeil SM, et al. Inactivation of the mouse Huntington's disease gene homolog Hdh. Science. 1995;269:407-10.

[8] Zeitlin S, Liu JP, Chapman DL, Papaioannou VE, Efstratiadis A. Increased apoptosis and early embryonic lethality in mice nullizygous for the Huntington's disease gene homologue. Nat Genet. 1995;11:155-63.

[9] White JK, Auerbach W, Duyao MP, Vonsattel JP, Gusella JF, Joyner AL, et al. Huntingtin is required for neurogenesis and is not impaired by the Huntington's disease CAG expansion. Nat Genet. 1997; 17:404-10.

[10] Dragatsis I, Levine MS, Zeitlin S. Inactivation of Hdh in the brain and testis results in progressive neurodegeneration and sterility in mice. Nat Genet. 2000;26:300-6.

[11] Kegel KB, Kim M, Sapp E, McIntyre C, Castano JG, Aronin N, et al. Huntingtin expression stimulates endosomallysosomal activity, endosome tubulation, and autophagy. J Neurosci. 2000;20:7268-78.

[12] Velier J, Kim M, Schwarz C, Kim TW, Sapp E, Chase K, et al. Wild-type and mutant huntingtins function in vesicle trafficking in the secretory and endocytic pathways. Exp Neurol. 1998;152:34-40.

[13] Aronin N, Chase K, Young C, Sapp E, Schwarz C, Matta $\mathrm{N}$, et al. CAG expansion affects the expression of mutant Huntingtin in the Huntington's disease brain. Neuron. 1995; 15:1193-201.

[14] Atwal RS, Xia J, Pinchev D, Taylor J, Epand RM, Truant R. Huntingtin has a membrane association signal that can modulate huntingtin aggregation, nuclear entry and toxicity. Hum Mol Genet. 2007; 16:2600-15.

[15] Hilditch-Maguire P, Trettel F, Passani LA, Auerbach A, Persichetti F, MacDonald ME. Huntingtin: An ironregulated protein essential for normal nuclear and perinuclear organelles. Hum Mol Genet. 2000;9:2789-97.

[16] Caviston JP, Holzbaur EL. Huntingtin as an essential integrator of intracellular vesicular trafficking. Trends Cell Biol. 2009;19:147-55.

[17] Strehlow AN, Li JZ, Myers RM. Wild-type huntingtin participates in protein trafficking between the Golgi and the extracellular space. Hum Mol Genet. 2007;16:391-409.

[18] Ritch JJ, Valencia A, Alexander J, Sapp E, Gatune L, Sangrey GR, et al. Multiple phenotypes in Huntington disease mouse neural stem cells. Mol Cell Neurosci. 2012;50:70-81.

[19] Myre MA, Lumsden AL, Thompson MN, Wasco W, MacDonald ME, Gusella JF. Deficiency of huntingtin has pleiotropic effects in the social amoeba Dictyostelium discoideum. PLoS Genet. 2011;7:e1002052.

[20] Wang Y, Steimle PA, Ren Y, Ross CA, Robinson DN, Egelhoff TT, et al. Dictyostelium huntingtin controls chemotaxis and cytokinesis through the regulation of myosin II phosphorylation. Mol Biol Cell. 2011;22:2270-81.

[21] Li X, Sapp E, Valencia A, Kegel KB, Qin ZH, Alexander J, et al. A function of huntingtin in guanine nucleotide exchange on Rab11. Neuroreport. 2008;19:1643-7.

[22] Jacobsen JC, Gregory GC, Woda JM, Thompson MN, Coser $\mathrm{KR}$, Murthy V, et al. HD CAG-correlated gene expression changes support a simple dominant gain of function. Hum Mol Genet. 2011;20:2846-60.

[23] Kegel KB, Sapp E, Yoder J, Cuiffo B, Sobin L, Kim YJ, et al. Huntingtin associates with acidic phospholipids at the plasma membrane. J Biol Chem. 2005;280:36464-73.

[24] Liu YF, Deth RC, Devys D. SH3 domain-dependent association of huntingtin with epidermal growth factor receptor signaling complexes. J Biol Chem. 1997;272:8121-4.

[25] Song C, Perides G, Liu YF. Expression of full-length polyglutamine-expanded Huntingtin disrupts growth factor receptor signaling in rat pheochromocytoma (PC12) cells. J Biol Chem. 2002;277:6703-7.

[26] Valenza M, Cattaneo E. Emerging roles for cholesterol in Huntington's disease. Trends Neurosci. 2011;34:474-86.

[27] Aubry L, Bugi A, Lefort N, Rousseau F, Peschanski M, Perrier AL. Striatal progenitors derived from human ES cells mature into DARPP32 neurons in vitro and in quinolinic acidlesioned rats. Proc Natl Acad Sci U S A. 2008;105:16707-12.

[28] Maglione V, Marchi P, Di Pardo A, Lingrell S, Horkey M, Tidmarsh E, et al. Impaired ganglioside metabolism in Huntington's disease and neuroprotective role of GM1. J Neurosci. 2010;30:4072-80.

[29] Chalon S. Omega-3 fatty acids and monoamine neurotransmission. Prostaglandins Leukot Essent Fatty Acids. 2006;75: 259-69.

[30] Vial D, Piomelli D. Dopamine D2 receptors potentiate arachidonate release via activation of cytosolic, arachidonatespecific phospholipase A2. J Neurochem. 1995;64:2765-72.

[31] Condray R, Yao JK. Cognition, dopamine and bioactive lipids in schizophrenia. Front Biosci (Schol Ed). 2011;3:298330.

[32] Lloyd KG, Davidson L. [3H]GABA binding in brains from Huntington's chorea patients: Altered regulation by phospholipids? Science. 1979;205:1147-9.

[33] Ellison DW, Beal MF, Martin JB. Phosphoethanolamine and ethanolamine are decreased in Alzheimer's disease and Huntington's disease. Brain Res. 1987;417:389-92. 
[34] Sakai T, Antoku Y, Iwashita H, Goto I, Nagamatsu K, Shii H. Chorea-acanthocytosis: Abnormal composition of covalently bound fatty acids of erythrocyte membrane proteins. Ann Neurol. 1991;29:664-9.

[35] Puri BK. Impaired phospholipid-related signal transduction in advanced Huntington's disease. Exp Physiol. 2001;86: 683-5.

[36] Glen AI, Cooper SJ, Rybakowski J, Vaddadi K, Brayshaw N, Horrobin DF. Membrane fatty acids, niacin flushing and clinical parameters. Prostaglandins Leukot Essent Fatty Acids. 1996;55:9-15.

[37] Morrow JD, Awad JA, Oates JA, Roberts LJ. 2nd. Identification of skin as a major site of prostaglandin D2 release following oral administration of niacin in humans. J Invest Dermatol. 1992;98:812-5.

[38] Block RC, Dorsey ER, Beck CA, Brenna JT, Shoulson I. Altered cholesterol and fatty acid metabolism in Huntington disease. J Clin Lipidol. 2010;4:17-23.

[39] Clifford JJ, Drago J, Natoli AL, Wong JY, Kinsella A, Waddington JL, et al. Essential fatty acids given from conception prevent topographies of motor deficit in a transgenic model of Huntington's disease. Neuroscience. 2002;109:818.

[40] Van Raamsdonk JM, Pearson J, Rogers DA, Lu G, Barakauskas VE, Barr AM, et al. Ethyl-EPA treatment improves motor dysfunction, but not neurodegeneration in the YAC128 mouse model of Huntington disease. Exp Neurol. 2005;196:266-72.

[41] Puri BK, Leavitt BR, Hayden MR, Ross CA, Rosenblatt A, Greenamyre JT, et al. Ethyl-EPA in Huntington disease: A double-blind, randomized, placebo-controlled trial. Neurology. 2005;65:286-92.

[42] Puri BK, Bydder GM, Manku MS, Clarke A, Waldman AD, Beckmann CF. Reduction in cerebral atrophy associated with ethyl-eicosapentaenoic acid treatment in patients with Huntington's disease. J Int Med Res. 2008;36:896-905.

[43] Randomized controlled trial of ethyl-eicosapentaenoic acid in Huntington disease: The TREND-HD study. Arch Neurol. 2008;65:1582-1589.

[44] Armstrong MJ, Miyasaki JM. Evidence-based guideline: Pharmacologic treatment of chorea in Huntington disease: Report of the guideline development subcommittee of the American Academy of Neurology. Neurology. 2012;79:597603.

[45] Sipione S, Rigamonti D, Valenza M, Zuccato C, Conti L, Pritchard J, et al. Early transcriptional profiles in huntingtininducible striatal cells by microarray analyses. Hum Mol Genet. 2002;11:1953-65.

[46] Tang TS, Tu H, Chan EY, Maximov A, Wang Z, Wellington $\mathrm{CL}$, et al. Huntingtin and huntingtin-associated protein 1 influence neuronal calcium signaling mediated by inositol- $(1,4,5)$ triphosphate receptor type 1. Neuron. 2003;39:227-39.

[47] Zhang N, Li B, Al-Ramahi I, Cong X, Held JM, Kim E, et al. Inhibition of lipid signaling enzyme diacylglycerol kinase epsilon attenuates mutant huntingtin toxicity. J Biol Chem. 2012;287:21204-13.

[48] Suopanki J, Gotz C, Lutsch G, Schiller J, Harjes P, Herrmann A, et al. Interaction of huntingtin fragments with brain membranes-clues to early dysfunction in Huntington's disease. J Neurochem. 2006;96:870-84.

[49] Kegel KB, Sapp E, Alexander J, Valencia A, Reeves P, Li X, et al. Polyglutamine expansion in huntingtin alters its interaction with phospholipids. J Neurochem. 2009;110:1585-97.

[50] Andrade MA, Bork P. HEAT repeats in the Huntington's disease protein. Nat Genet. 1995;11:115-6.
[51] Michalek M, Salnikov ES, Werten S, Bechinger B. Membrane interactions of the amphipathic amino terminus of huntingtin. Biochemistry. 2013;52:847-58.

[52] Burke KA, Kauffman KJ, Umbaugh CS, Frey SL, Legleiter J. The interaction of polyglutamine peptides with lipid membranes is regulated by flanking sequences associated with huntingtin. J Biol Chem. 2013;288:14993-5005.

[53] Rockabrand E, Slepko N, Pantalone A, Nukala VN, Kazantsev A, Marsh JL, et al. The first 17 amino acids of Huntingtin modulate its sub-cellular localization, aggregation and effects on calcium homeostasis. Hum Mol Genet. 2007;16:61-77.

[54] Michalek M, Salnikov ES, Bechinger B. Structure and Topology of the Huntingtin 1-17 Membrane Anchor by a Combined Solution and Solid-State NMR Approach. Biophys J. 2013;105:699-710.

[55] Kim MW, Chelliah Y, Kim SW, Otwinowski Z, Bezprozvanny I. Secondary structure of Huntingtin amino-terminal region. Structure. 2009; 17:1205-12.

[56] Kegel KB, Schewkunow V, Sapp E, Masso N, Wanker EE, DiFiglia $M$, et al. Polyglutamine expansion in huntingtin increases its insertion into lipid bilayers. Biochem Biophys Res Commun. 2009;387:472-5.

[57] Maiuri T, Woloshansky T, Xia J, Truant R. The huntingtin N17 domain is a multifunctional CRM1 and Ran-dependent nuclear and cilial export signal. Hum Mol Genet. 2013;22:1383-94.

[58] Gu X, Greiner ER, Mishra R, Kodali R, Osmand A, Finkbeiner $\mathrm{S}$, et al. Serines 13 and 16 are critical determinants of fulllength human mutant huntingtin induced disease pathogenesis in HD mice. Neuron. 2009;64:828-40.

[59] Lemmon MA. Phosphoinositide recognition domains. Traffic. 2003;4:201-13.

[60] Di Paolo G, De Camilli P. Phosphoinositides in cell regulation and membrane dynamics. Nature. 2006;443:651-7.

[61] Kutateladze TG. Translation of the phosphoinositide code by PI effectors. Nat Chem Biol. 2010;6:507-13.

[62] Rameh LE, Cantley LC. The role of phosphoinositide 3-kinase lipid products in cell function. J Biol Chem. 1999;274:834750.

[63] Vanhaesebroeck B, Waterfield MD. Signaling by distinct classes of phosphoinositide 3-kinases. Exp Cell Res. 1999;253:239-54.

[64] Schlessinger J. Cell signaling by receptor tyrosine kinases. Cell. 2000;103:211-25.

[65] Kalchman MA, Koide HB, McCutcheon K, Graham RK, Nichol K, Nishiyama K, et al. HIP1. a human homologue of S. cerevisiae Sla2p, interacts with membrane-associated huntingtin in the brain. Nat Genet. 1997;16:44-53.

[66] Waelter S, Scherzinger E, Hasenbank R, Nordhoff E, Lurz $\mathrm{R}$, Goehler $\mathrm{H}$, et al. The huntingtin interacting protein HIP1 is a clathrin and alpha-adaptin-binding protein involved in receptor-mediated endocytosis. Hum Mol Genet. 2001;10:1807-17.

[67] Hyun TS, Rao DS, Saint-Dic D, Michael LE, Kumar PD, Bradley SV, et al. HIP1. HIP1r stabilize receptor tyrosine kinases and bind 3-phosphoinositides via epsin N-terminal homology domains. J Biol Chem. 2004;279:14294-306.

[68] Yanai A, Huang K, Kang R, Singaraja RR, Arstikaitis P, Gan $\mathrm{L}$, et al. Palmitoylation of huntingtin by HIP14 is essential for its trafficking and function. Nat Neurosci. 2006;9: 824-31.

[69] Young FB, Butland SL, Sanders SS, Sutton LM, Hayden MR. Putting proteins in their place: Palmitoylation in Huntington disease and other neuropsychiatric diseases. Prog Neurobiol. 97:220-238 
[70] Aiken CT, Steffan JS, Guerrero CM, Khashwji H, Lukacsovich T, Simmons D, et al. Phosphorylation of threonine 3: Implications for Huntingtin aggregation and neurotoxicity. J Biol Chem. 2009;284:29427-36.

[71] Atwal JK, Chen Y, Chiu C, Mortensen DL, Meilandt WJ, Liu Y, et al. A therapeutic antibody targeting BACE1 inhibits amyloid-beta production in vivo. Sci Transl Med. 2011;3:84ra43.

[72] Steffan JS, Agrawal N, Pallos J, Rockabrand E, Trotman LC, Slepko N, et al. SUMO modification of Huntingtin and Huntington's disease pathology. Science. 2004;304:100-4.

[73] Thompson LM, Aiken CT, Kaltenbach LS, Agrawal N, Illes $\mathrm{K}$, Khoshnan A, et al. IKK phosphorylates Huntingtin and targets it for degradation by the proteasome and lysosome. J Cell Biol. 2009; 187:1083-99.

[74] Humbert S, Bryson EA, Cordelieres FP, Connors NC, Datta $\mathrm{SR}$, Finkbeiner S, et al. The IGF-1/Akt pathway is neuroprotective in Huntington's disease and involves Huntingtin phosphorylation by Akt. Dev Cell. 2002;2:831-7.

[75] Luo S, Vacher C, Davies JE, Rubinsztein DC. Cdk5 phosphorylation of huntingtin reduces its cleavage by caspases: Implications for mutant huntingtin toxicity. J Cell Biol. 2005;169:647-56.

[76] Warby SC, Chan EY, Metzler M, Gan L, Singaraja RR, Crocker SF, et al. Huntingtin phosphorylation on serine 421 is significantly reduced in the striatum and by polyglutamine expansion in vivo. Hum Mol Genet. 2005;14:1569-77.
[77] Anne SL, Saudou F, Humbert S. Phosphorylation of huntingtin by cyclin-dependent kinase 5 is induced by DNA damage and regulates wild-type and mutant huntingtin toxicity in neurons. J Neurosci. 2007;27:7318-28.

[78] Jeong H, Then F, Melia TJ, Jr, Mazzulli JR, Cui L, Savas JN, et al. Acetylation targets mutant huntingtin to autophagosomes for degradation. Cell. 2009;137:60-72

[79] Kagan BL, Hirakura Y, Azimov R, Azimova R. The channel hypothesis of Huntington's disease. Brain Res Bull. 2001;56:281-4.

[80] Valencia A, Reeves PB, Sapp E, Li X, Alexander J, Kegel $\mathrm{KB}$, et al. Mutant huntingtin and glycogen synthase kinase 3beta accumulate in neuronal lipid rafts of a presymptomatic knock-in mouse model of Huntington's disease. J Neurosci Res. 2010;88:179-90.

[81] Takano H, Gusella JF. The predominantly HEAT-like motif structure of huntingtin and its association and coincident nuclear entry with dorsal, an NF-kB/Rel/dorsal family transcription factor. BMC Neurosci. 2002;3:15.

[82] Hennig L. WinGene/WinPep: User-friendly software for the analysis of amino acid sequences. Biotechniques. 1999;26:1170-2.

[83] Guex N, Peitsch MC, SWISS-MODEL the Swiss-PdbViewer: An environment for comparative protein modeling. Electrophoresis. 1997; 18:2714-23. 